\title{
ADJUSTABLE ANTENNA USING PLASMA MEDIUM
}

\author{
N. A. N. ROSLAN ${ }^{1}$, A. N. DAGANG ${ }^{1 *}$ AND R. UMAR ${ }^{2}$ \\ ${ }^{1}$ Faculty of Ocean Engineering Technology and Informatics, Universiti Malaysia Terengganu, 21030 Kuala Nerus, \\ Terengganu, Malaysia. ${ }^{2}$ East Coast Environmental Research Institute, Universiti Sultan Zainal Abidin, 21030 Kuala Nerus, \\ Terengganu, Malaysia.
}

*Corresponding author: nazri.dagang@umt.edu.my

http://doi.org/10.46754/umtjur.2021.10.003

\begin{abstract}
Plasma antenna is a type of radio antenna in which plasma is used instead of the metal elements of a traditional antenna. One of the advantages of using plasma antenna is it can improve conventional devices in terms of switching (on/off) the antenna using plasma generator, which could make it invisible to the radar. In addition, when antenna conductivity can be controlled through manipulation of plasma parameters, an adjustable antenna can be designed. The aims of this research are to design and simulate plasma antenna using CST software, and subsequently determine the characteristics of plasma antenna with different neon pressure applied (10, 15, 20 Torr). The custommade cylindrical discharge tube shape with the length and diameter of $160 \mathrm{~mm}$ and $10 \mathrm{~mm}$ was used. The research focuses on simulation in obtaining antenna parameters while conducting an experimental work to obtain electrical parameters which are needed in calculating the plasma parameters. The discharge tube shape was designed and simulated using Computer Simulation Technology (CST) that can generate antenna parameters such as return loss, gain, directivity and radiation pattern. The simulation result shows the radiation pattern of discharge tube as omni-directional and it gives neon discharge at 20 Torr that has the best performance in return loss at $-24.490250 \mathrm{~dB}$, compared to neon at 15 and 10 Torr which have lower values at $-21.578 \mathrm{~dB}$ and $-13.901 \mathrm{~dB}$ respectively. In terms of gain and directivity, neon at 10 Torr has the highest value, which is $2.688 \mathrm{~dB}$ and $5.437 \mathrm{dBi}$, respectively. From the results, it can be concluded that when gas pressure is increased, the antenna gains decreases. Neon discharge at a middle pressure of 15 Torr is considered to provide a good antenna gain and give effective signal transmission compared to lower pressure of 15 and higher pressure of 20 Torr.
\end{abstract}

Keywords: Plasma, antenna, adjustable antenna, CST, neon.

\section{Introduction}

A plasma antenna is a type of radio antenna in which plasma is used instead of the metal elements of a conventional antenna. A plasma uses ionized gas as a conducting medium instead of a metal to receive and transmit a signal and this idea or concept is from J. Hettinger in 1919 (Madda, 2017). Highly ionized plasma is a good conductor and it is can be used as a transmission line where the surface wave propagates along the plasma column. Radio waves are important as they conquer the information in telecommunication technology and has the longest wavelength in the spectrum. Nowadays, it will be difficult to create new technology without radio waves as it is a good delivery service (Lucas, 2015).
Computer Simulation Technology Microwave Studio (CST MWS) software based on the finite integral technique is used to simulate the performance of a cylindrical plasma monopole antenna. In this software, the plasma behavior is given by the model of drude dispersion. The drude dispersion model defines simple characteristics of a collective of free positive and negative charge carriers conducting electrically, where the thermal movement of electrons is neglected ( $\mathrm{Li}$ et al., 2011).

According to Desire (2018), in 1994 Paul Drude developed the Drude model to describe the movement of electrons in materials. The Drude model assumes, based on the kinetic theory of electrons, that the material is produced by fixed positive ions and non-interacting electron gas. Also known as Drude parameters are the plasma frequency and collision frequency. 
There are two plasma characteristics used in this research, plasma frequency and collision frequency. The plasma frequency defines a natural frequency of the plasma and it measures the amount of ionization in plasma. The collision frequency that occurs in gases is important in radio frequency field (Ja'afar et al., 2018). The values of plasma and collision frequency are used as input parameters in the CST software for designing the plasma antenna.

In recent years, plasma antenna remains an extensive trend in the research of antenna than traditional metal antenna (Bonde et al., 2014). The disadvantages of the metal antenna are less efficient because the design of metal antenna is limited to its length, which affects its signal strength, and its higher weight compared to plasma antenna. It also has a ringing effect, where energy is wasted and unwanted electromagnetic (EM) waves are emitted (Bhavarthe et al., 2015).

The advantages of plasma antenna are invisible to radar system. There is no ringing effect and it can be designed to be smaller, lighter and more efficient. It makes metal conductors more portable by replacing with semi-conductor chips or gasses (Shriwas \& Gulhane, 2015). There is no "ringing" effect in plasma antenna, since it allows extremely short pulses. Antenna ringing is undesirable as it corrupts the transmitted pulse waveform and reduces range resolution. Study on cylindrical plasma antenna, when the plasma parameters were adjusted, shows a good agreement (Barro et al., 2018). An interesting study on plasma parameters effect on antenna performance was reported where the plasma parameters were measured using microwave interferometry method (Ghaderi et al., 2019). Thus, it is important to investigate the performance of plasma antenna when plasma parameters are changed. The plasma antenna to be used is cylindrical type and is custom made discharge tube containing neon gas. It is capable of replacing the traditional metal antenna and it capable of transmitting and receiving radio signal strength.

A discharge tube was prepared with plasma containing both neon ions and many neutral atoms. The AC voltage was applied across at both ends of a discharge tube and high voltage between electrodes cause electrons to flow. They become electrically conductive when the plasma antenna tubes were energized, and could transmit and receive radio signals. When de-energized, these return to non-conducting elements and have failed to show radio signals being tested.

This research focuses on how the adjustable values, namely gas pressure and electrical properties, can affect the antenna performance in terms of its return loss, resonance frequency, gain, directivity and signal strength.

\section{Materials and Methods}

\section{Discharge Tube}

The material used in this research was the same length and diameter of custom-made discharge tubes which is $10 \mathrm{~mm}$ and $160 \mathrm{~mm}$ as shown in Figure 1. Different pressure was applied for each tube (10 Torr, 15 Torr and 20 Torr) and it had the same function as the plasma antenna. High voltage was required to light up the discharge tube. The DC power supply was connected to the Cold Cathode Fluorescent Lamp (CCFL) inverter, which transformed Direct Current (DC) voltage to Alternating Current (AC) voltage and increased the voltage. CCFL inverter was connected to both ends of discharge tube. 


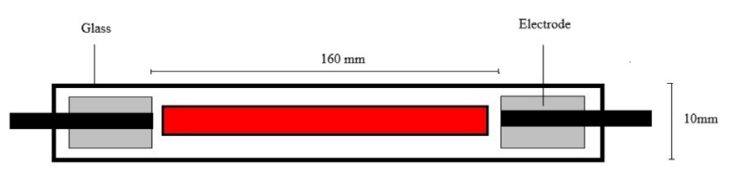

Figure 1: Diagram of discharge tube with length and diameter

\section{Experimental Setup}

High voltage was required to light up the discharge tube. The DC power supply was connected to the CCFL inverter, which transforms $\mathrm{DC}$ voltage to $\mathrm{AC}$ voltage and increases the voltage. CCFL inverter was connected to both ends of the discharge tube. The connection for the circuit as shown in Figure 2 below where electrical properties measurement was done by reading the current and voltage values by using a digital oscilloscope. In addition, high voltage probe, current probe, and amplifier were also connected according to the connection shown in Figure 2. Electrical property measurement was done by reading the current and voltage values by using a digital oscilloscope. In addition, high voltage probe, current probe, and amplifier were also connected according to the connection shown in Figure 3 below.

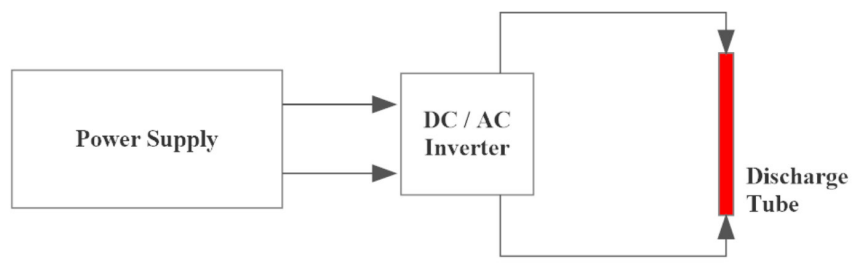

Figure 2: Experimental set-up of discharge tube

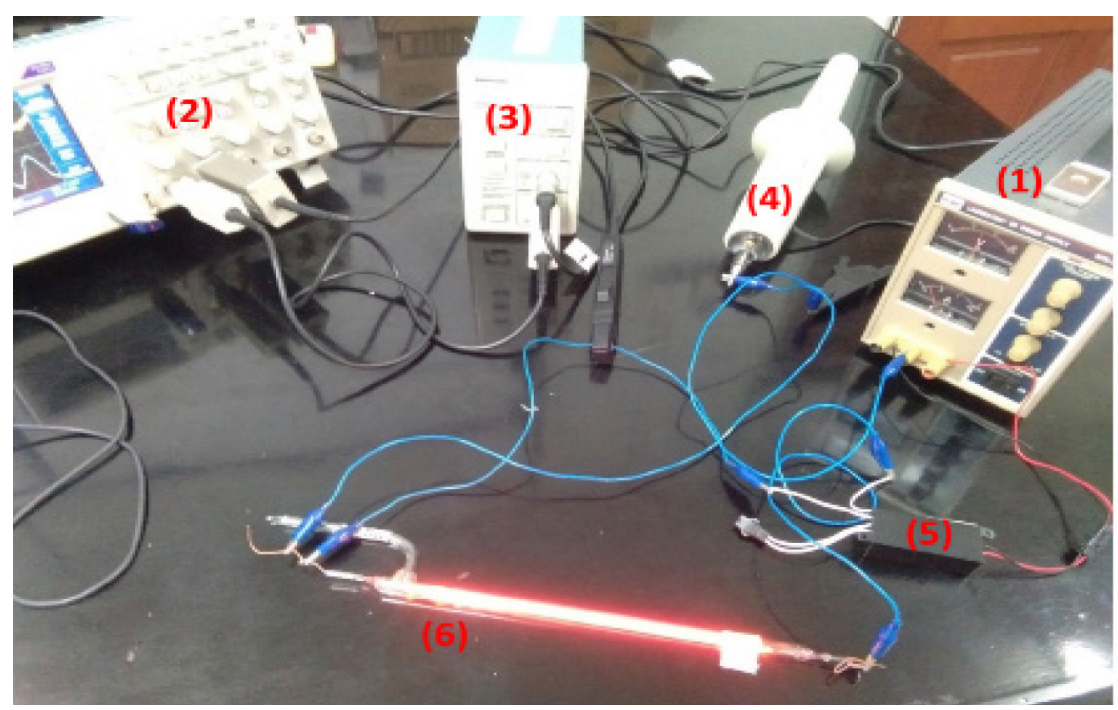

Figure 3: Setup for electrical properties measurement where (1) DC power supply, (2) Digital oscilloscope, (3) High current probe and its amplifier, (4) High voltage probe, (5) CCFL inverter, (6) Discharge tube 


\section{Glomac Programming}

The value of the current and voltage from electrical properties measurement and physical parameters of the discharge tube, such as tube radius, gas pressure and gas fill temperature was inserted into Glomac programming. Glomac is a numerical model that can generate electron temperature, average gas density and average electron density (Lister \& Coe, 1993). Electron temperature and density generated by Glomac were used to calculate theoretically the plasma frequency and collision frequency.

\section{Plasma Parameters Calculation}

The Glomac programming generated the value of electron temperature, average electron density, and average gas density. These values were used to calculate the plasma frequency and collision frequency. Plasma material was not found in the material library in CST software. Therefore, the values of plasma frequency and collision frequency were calculated using Equations 1 and 2 respectively. From the calculation, plasma frequency and collision frequency values were used as input parameters in the CST software for design plasma antenna. Thus, the value of gas density (n) used to calculate the collision frequency using the ideal gas equation below:

$$
n=\frac{P}{k T}
$$

where $P$ is the value of the gas pressure, $k$ is the value of Boltzmann constant is equaled to $1.381 \times 10^{-23} \mathrm{JK}^{-1}$ and $T$ is the value of gas temperature (at room temperature $=300 \mathrm{~K}$ ). Next, to calculate the value of electron speed $\left(v_{\mathrm{e}}\right)$, the equation for the average kinetic energy of a number of gas molecules that were given at temperature as shown in equations below:

$$
\frac{1}{2} m_{e} v_{e}^{2}=\frac{3}{2} k T
$$

Here $m_{\mathrm{e}}$ is electron mass at $9.109 \times 10^{-31} \mathrm{~kg}$. The value of $v_{\mathrm{e}}$, electron speed is calculated, by arranging from Equation 2, and Equation 3 can be obtained.

$$
v_{\mathrm{e}}=\sqrt{\frac{3 k T_{\mathrm{e}}}{m_{\mathrm{e}}}}
$$

where $k$ is Boltzman's constant, $1.381 \times 10^{-23}$ $\mathrm{JK}^{-1}$ and $T_{\mathrm{e}}$ is electron temperature in Kelvin (obtained from GLOMAC). The value of the collision cross section $\sigma$ is calculated using Equation 4 as shown below:

$$
\sigma=\left(2.87 \times 10^{-17}\right)(P) \mathrm{cm}^{2}
$$

where $P_{\mathrm{c}}$ is the probability of collision which is obtained by using literature (Lieberman \& Lishtenberg, 2005).

Plasma frequency, $\omega_{\mathrm{p}}$, can be calculated by using Equation 5 ( $n_{\mathrm{e}}$ is the density of the ionized electrons, where $e$ is electron charge), while collision frequency, $v_{\mathrm{c}}$, can be obtained using Equation 6. The plasma frequency is based on the electron number density and collision frequency relates to the electron temperature, those obtained from Glomac programming. From the calculation, the values of plasma frequency and collision frequency were obtained and used as input parameters in CST software for plasma antenna.

$$
\begin{gathered}
\omega_{p}=\sqrt{\frac{e^{2} n_{e}}{\varepsilon_{0} m}} \\
v_{c}=n\left\langle\sigma v_{e}\right\rangle
\end{gathered}
$$

\section{Simulation of Plasma Antenna}

CST Studio Suite 2018 software can be accomplished to design a plasma antenna. The design was based on the length and diameter of the discharge tube and the values of plasma frequency and collision frequency were calculated by the previous step. The type of antenna designed via CST software was a monopole antenna to the presence of an actual shaped discharge tube. It was simulated using copper as a coupling sleeve to obtain antenna parameters. Design for neon tube as a plasma antenna is shown in Figure 4. The signal can be transmitted or received using coupling sleeve 
(middle of the tube) connected to the SMA connector. The coupling sleeve acts as an input terminal which is used to connect the fluorescent tube with external signals and measuring equipment. In addition, the frequency is focused in the range of $1 \mathrm{GHz}$ to $10 \mathrm{GHz}$ for all designs in this work.

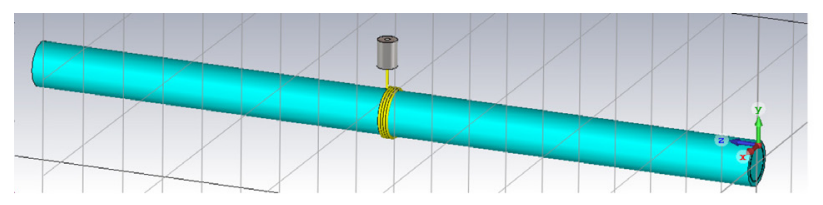

Figure 4: Design of plasma antenna using CST

\section{Results and Discussion}

\section{Electrical Properties Measurement}

When the discharge tube was ionized, it would light up using DC power supply connected to the CCFL inverter, and the DC supply was tuned until the maximum visible light was obtained. As a result, the current was maintained at low levels so as to establish the stable value of the discharge current via the digital oscilloscope. Table 1 shows the value of discharge current and discharge voltage recorded. The discharge tube was lighted up between $320 \mathrm{~V}$ and $340 \mathrm{~V}$ of the voltage supply and released $38 \mathrm{~mA}$ of discharge current for 10 Torr plasma discharge tube, $43 \mathrm{~mA}$ for 15 Torr, and for $39 \mathrm{~mA}$ for 20 Torr plasma discharge tubes. In the experiment, the higher the voltage, the higher the current flow was because the lamp brightness depends on the current and voltage. The amount of current required to light up the lamp was excessive and caused the electrons to produce extra heat (Dagang et al., 2017). Figure 5 shows the output voltage and current which appeared on the digital oscilloscope.

Table 1: The value of discharge current and discharge voltage

\begin{tabular}{ccc}
\hline Pressure (Torr) & Current (A) & Voltage (V) \\
\hline 10 & 0.038 & 320 \\
15 & 0.043 & 336 \\
20 & 0.039 & 340 \\
\hline
\end{tabular}

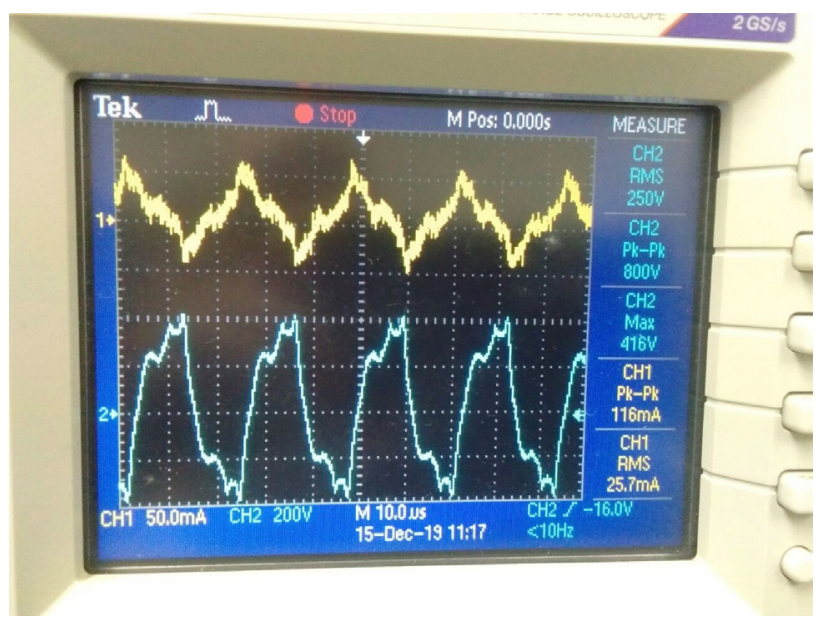

Figure 5: The output voltage and current appeared on the digital oscilloscope 


\section{Plasma Parameters Calculation}

The result of discharge current from electrical properties measurement and other properties such as tube radius, gas fill temperature and gas pressure were put into Glomac programming to calculate the plasma parameters. Glomac programming generated the output data as shown in Table 2 and for the different gas pressure it is found that the electron temperature decreases from $5572.8 \mathrm{~K}$ to $5543.2 \mathrm{~K}$. It shows that slight changing was due to the changes of the gas pressure. According to (Shah et al., 2008), the electron temperature was found to decrease with an increase in filling pressure. It explains that as the nitrogen gas pressure in the chamber increases, the number of collisions between the electron and the plasma species increases. Hence, the gas particle increased the gas temperature and electron temperature decreased. The electron temperature decreases when the pressure is increased from 10 Torr to 20 Torr.
Table 2 also shows the results of average electron density increases by increasing the gas pressure inside the plasma discharge tube. For an increase of gas pressure inside the discharge tube, there will be more atom, molecule and free electron. Hence, a lot of collisions occurred between those particles. This caused the production of many free electrons and ions, thus increasing the electron density. The effect of the gas flow rate and filling pressure on the electron density proved that the electron density decreased with an increase in the gas flow rate and filling pressure (Qayyum et al., 2003). The value of average electron density and electron temperature was inserted into Equations 5 and 6 . The result of plasma frequency and collision frequency is shown in Table 3. The gas pressure at 20 Torr had plasma frequency of $6.049 \times 1010$ $\mathrm{rad} / \mathrm{s}$ which is the highest compared with the gas pressure at 10 Torr with $5.206 \times 1010 \mathrm{rad} / \mathrm{s}$, and the gas pressure at 15 Torr with $5.518 \times 1010$ $\mathrm{rad} / \mathrm{s}$. When the electron density increased, the plasma frequency also increased.

Table 2: The results generated from Glomac programming

\begin{tabular}{ccc}
\hline \multirow{2}{*}{ Pressure (Torr) } & $\begin{array}{c}\text { Average Electron Density } \\
n_{e}\left(\mathbf{m}^{-3}\right)\end{array}$ & $\begin{array}{c}\text { Electron Temperature } \\
T_{e}(\mathbf{K}, \mathbf{e V})\end{array}$ \\
\hline 10 & $8.52 \times 10^{17}$ & $5572.8,0.480$ \\
15 & $9.57 \times 10^{17}$ & $5553.9,0.479$ \\
20 & $1.15 \times 10^{18}$ & $5543.2,0.478$ \\
\hline
\end{tabular}

Table 3: Plasma frequency and collision frequency of different gas pressure

\begin{tabular}{ccc}
\hline Pressure (Torr) & $\begin{array}{c}\text { Plasma Frequency } \\
\mathbf{W}_{p}\left(\mathbf{1 0}^{\mathbf{1 0}}, \mathbf{R a d i a n} / \mathbf{s}\right)\end{array}$ & $\begin{array}{c}\text { Collision Frequency } \\
\boldsymbol{v}_{c}\left(\mathbf{1 0}^{\mathbf{9}}, \mathbf{s}^{-1}\right)\end{array}$ \\
\hline 10 & 5.206 & 1.395 \\
15 & 5.518 & 2.080 \\
20 & 6.049 & 2.780 \\
\hline
\end{tabular}

The collision frequency was the highest in gas pressure at 20 Torr of $2.780 \times 10^{9} \mathrm{~s}^{-1}$ followed by gas pressure at 15 Torr of $2.080 \mathrm{x}$ $10^{9} \mathrm{~s}^{-1}$ and gas pressure at 10 Torr of $1.395 \mathrm{x}$ $10^{9} \mathrm{~s}^{-1}$. The collision frequency increases with the increase of gas pressure. This might be due to higher electron density which made shorter mean free path and consequently produced more collisions. Plasma frequency and collision frequency values for different gas pressure were applied to the plasma properties in CST design.

\section{Antenna Simulation Results}

The $S_{11}$ parameter indicates how much power antenna has to reflect on a certain frequency. Any frequency at which $S_{11}$ has a minimum value is called antenna resonant frequency, and at 
that frequency antenna will transmit maximum power (Kumar, 2018). The graph in Figure 6 shows the resonant frequency for the different pressure plasma. In detail, the return loss for 10 Torr plasma discharge tube is $-13.900794 \mathrm{~dB}$ at frequency $4.168 \mathrm{GHz}$, for 15 Torr is -21.578111 $\mathrm{dB}$ at frequency $4.276 \mathrm{GHz}$ and for 20 Torr is $-24.490250 \mathrm{~dB}$ at frequency $4.330 \mathrm{GHz}$. This shows that the gas pressure influenced the return loss of plasma antenna. When the gas pressure was increased, the bandwidth turned wider with the return loss getting better. The return loss changed approximately from $-14 \mathrm{~dB}$ to $-25 \mathrm{~dB}$ when gas pressure was increased. Figures 7 to 9 show the frequency range for each plasma antenna with different gas pressures. The range frequency for gas pressure of 10, 15 and 20 Torr is considerably the same as they are between 3.9234 GHz and 4.7596 GHz. This frequency range is mainly used for communication and satellite broadcast.

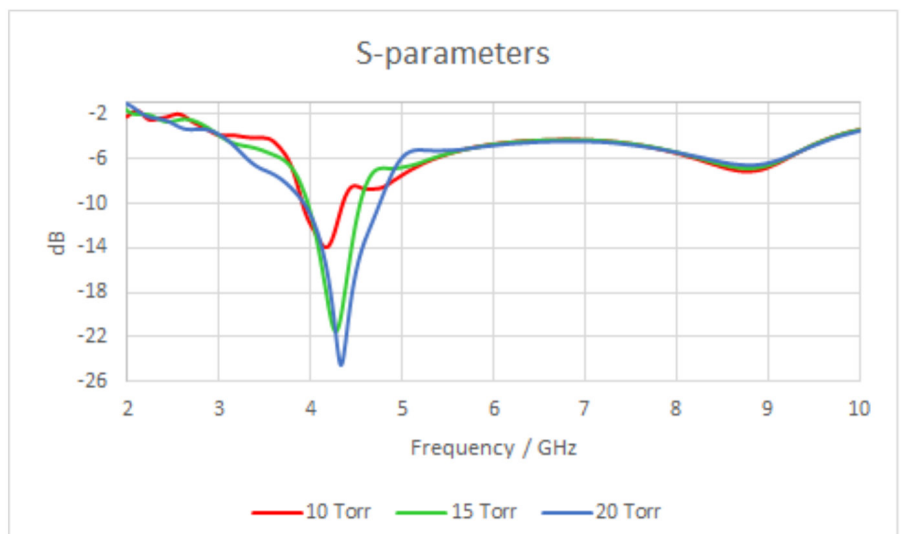

Figure 6: The graph of return loss, $\left(S_{11}\right)$ results for different gas pressure of plasma discharge tube

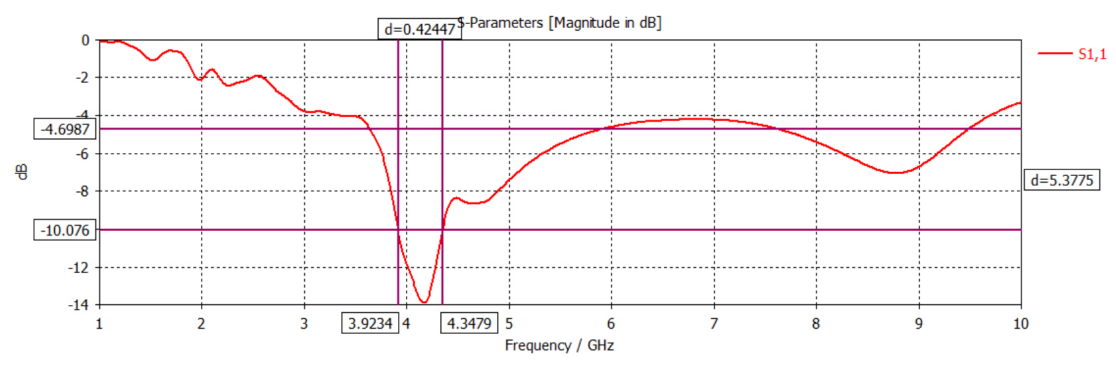

Figure 7: The graph of range frequency for gas pressure 10 Torr between $3.9234 \mathrm{GHz}$ and $4.3479 \mathrm{GHz}$

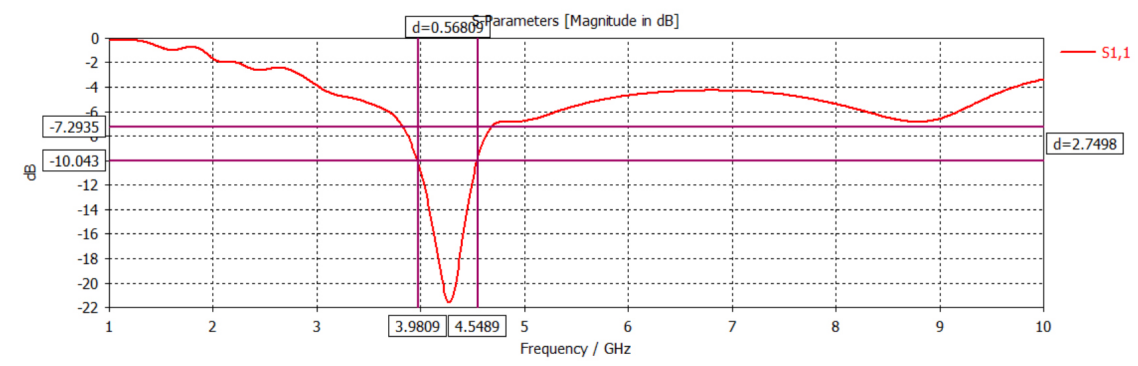

Figure 8: The graph of range frequency for gas pressure 15 Torr between $3.9809 \mathrm{GHz}$ and $4.5489 \mathrm{GHz}$ 


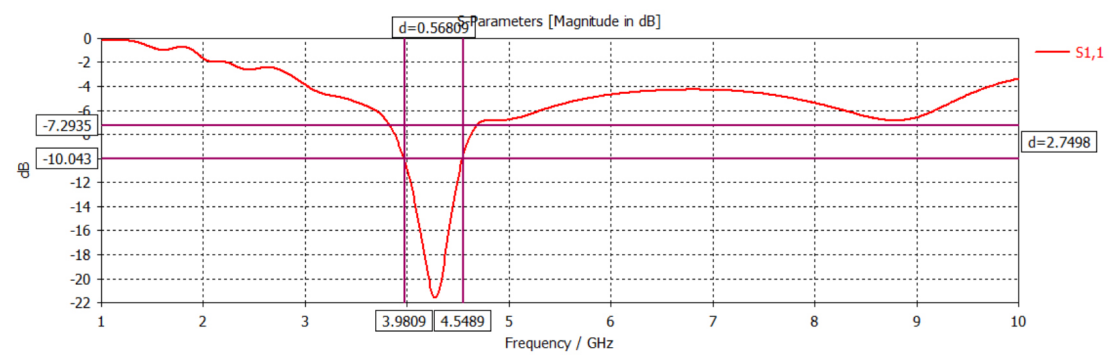

Figure 9: The graph of range frequency for gas pressure 20 Torr between $3.933 \mathrm{GHz}$ and $4.7596 \mathrm{GHz}$

The plasma parameter such as resonant frequency, return loss, gain and directivity acquired from the simulation is shown in Table 4. The resonant frequencies can be seen to have slightly shifted accordingly when pressure was increased. The antenna gain generated by 10 Torr is higher than 15 Torr and 20 Torr. For plasma discharge tube 10 Torr, the value gain and directivity acquired are $2.688 \mathrm{~dB}$ and 5.437 , for 15 Torr are $2.158 \mathrm{~dB}$ and $4.753 \mathrm{dBi}$, and for 20 Torr are $1.987 \mathrm{~dB}$ and $4.795 \mathrm{dBi}$, respectively. The gain determined how well the antenna can change the input power to radio waves.
The results show that gas pressure at 10 Torr of plasma discharge tube is good for antenna gain and the most effective antenna to deliver signals compared to those at 15 and 20 Torr. It can be said that for all different gas pressures, when the pressure increased, the antenna gain decreased. As the collision frequency increased when pressure is increased, plasma conductivity decreased. This will influence the antenna gain (Ja'afar et al., 2014). Therefore, it can be concluded that the change of collision frequency due to the changes in gas pressure can influence the antenna gain.

Table 4: The $S_{11}$, resonant frequency, gain and directivity for different gas pressure

\begin{tabular}{ccccc}
\hline Pressure (Torr) & Frequency $(\mathbf{G H z})$ & Return Loss $\boldsymbol{S}_{\mathbf{1 1}} \mathbf{( d B )}$ & Gain $(\mathbf{d B})$ & Directivity (dBi) \\
\hline 10 & 4.168 & -13.901 & 2.688 & 5.437 \\
15 & 4.276 & -21.578 & 2.158 & 4.753 \\
20 & 4.330 & -24.490 & 1.987 & 4.795 \\
\hline
\end{tabular}

Figure 10 presents polar pattern of radiation pattern in terms E-plane $\left(\mathrm{phi}=90^{\circ}\right.$ ) for three types of gas pressure. In the far field area, the radiation pattern is determined and represented as a function of the directional coordinates (Ja'afar et al., 2014). Figures 11 to 13 show the radiation pattern in polar for different gas pressure of plasma discharge tube. The radiation pattern for gas pressure 10 Torr has the main lobe magnitude of $2.7 \mathrm{~dB}$ at $55.9^{\circ}$ while for gas pressure 15 Torr has the main lobe magnitude of $2.17 \mathrm{~dB}$ at $58.9^{\circ}$ and gas pressure 20 Torr has the main lobe magnitude of $2 \mathrm{~dB}$ at $58.3^{\circ} \mathrm{FF}$. 


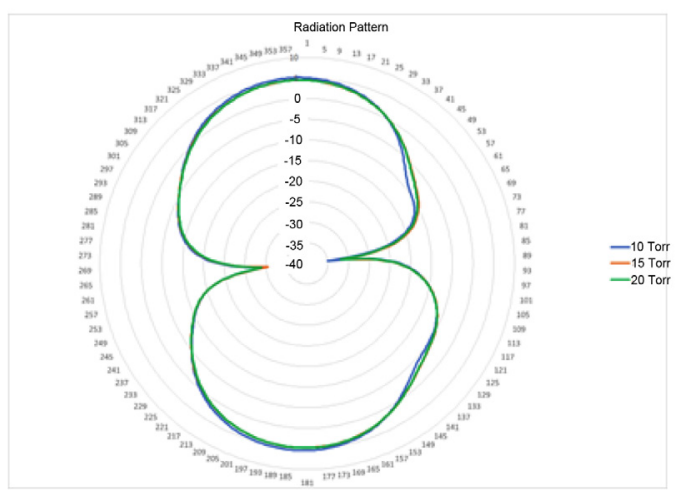

Figure 10: The radiation pattern for different gas pressure of plasma discharge tube

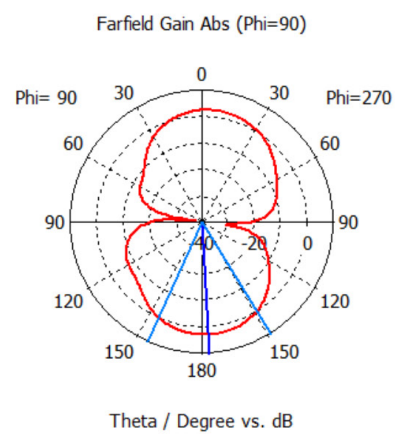

(a)

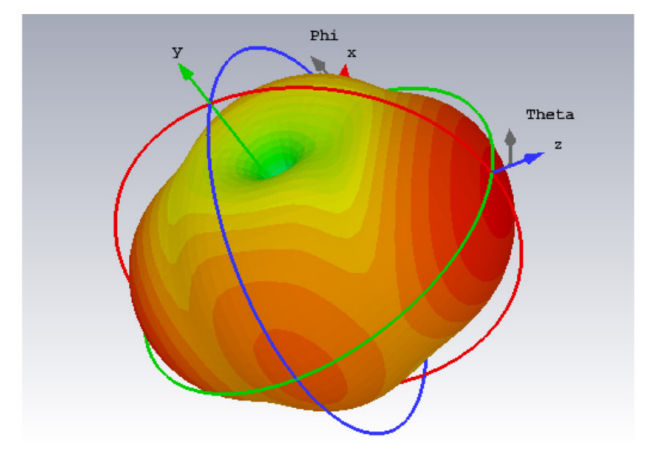

(b)

Figure 11: The radiation pattern for gas pressure 10 Torr (a) polar pattern (b) 3-dimension pattern Farfield Gain Abs (Phi=90)

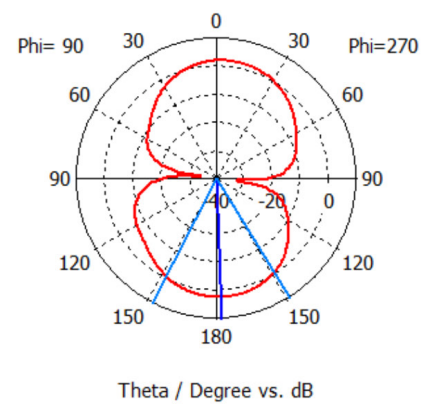

(a)

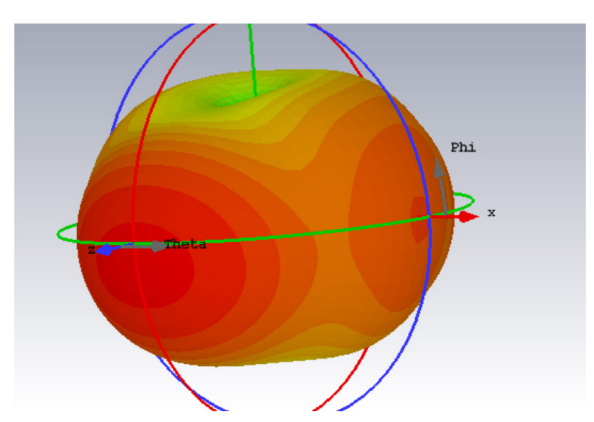

(b)

Figure 12: The radiation pattern for gas pressure 15 Torr (a) polar pattern (b) 3-dimension pattern 


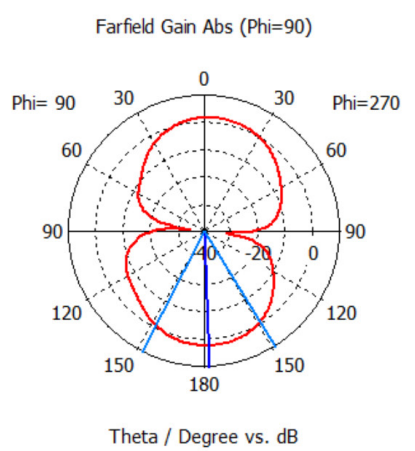

(a)

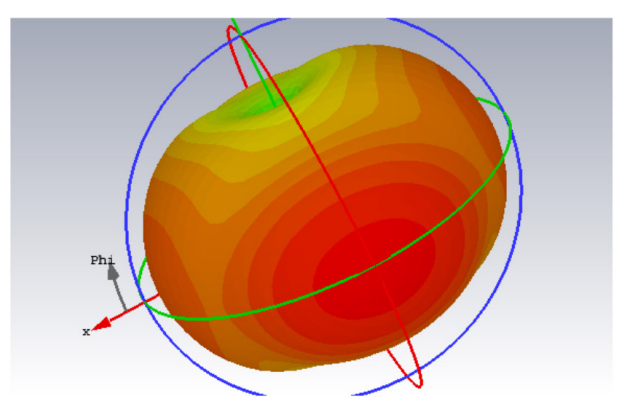

(b)

Figure 13: The radiation pattern for gas pressure 20 Torr (a) polar pattern (b) 3-dimension pattern

The results show that monopole plasma antenna radiation patterns using neon gas with different pressure as a plasma medium looks similar to each other. However, the main lobe magnitude shows the gas pressure 10 Torr of plasma discharge tube is higher than 15 and 20 Torr. It shows the radiation pattern of plasma antenna used which is omnidirectional, shown by the circular pattern of the main lobe on both sides of a plane. An omnidirectional antenna has no specific directivity in a given place. It is important to acknowledge the radiation pattern of an antenna since the antenna can pick up more signals accurately. Since the radiation pattern for the antenna constructed is omnidirectional, the plasma antenna is able to receive in every direction disregarding its position either vertical or horizontal.

\section{Conclusion}

The calculation of plasma parameters, such as electron temperature and electron density, is essential to design the plasma antenna. The selection of electrical properties is important for the design of the plasma antenna in CST. The electron temperature is found to decrease with an increase in filling pressure. Besides, the electron density decreases with an increase of the gas pressure. The plasma properties can be calculated by the plasma frequency and the collision frequency. When the electron density increases, the plasma frequency also increases thus the collision frequency increases with the gas pressure. In addition, this type of adjustable (pressure, electrical properties) monopole plasma antenna design gives omnidirectional radiation pattern. This radiation pattern is good where it could transmit and receive the signals from many directions. Plasma antenna provides best characteristics as it offers the optimum values for the main antenna parameters. This is due to the values of plasma parameters which are moderate compared to 10 Torr and 20 Torr.

\section{Acknowledgements}

The authors would like to express gratitude to the Faculty of Ocean Engineering Technology and Informatics, Universiti Malaysia Terengganu for providing research facilities as well as supporting staff for this research project.

\section{References}

Barro, O. A., Himdi, M., \& Lafond, O., (2018). Reconfigurable cylindrical plasma antenna. Progress in Electromagnetic Research, 66, 65-72.

Bhavarthe, P., Govekar, L., \& Tyagi, P. (2015). Comparative study of Metal Antenna \& Plasma Antenna. International Journal of Innovative Research in Electrical, Electronics, Instrumentation and Control Engineering, 3(1), 129-131. 
Bonde, S., Ghiye, V., \& Dhande, A. (2014). A study of plasma antenna parameters with different gases. Proceedings 4th International Conference on Communication Systems and Network Technologies, CSNT 2014, (2), 16-19.

Dagang, A. N., Karunamurthy, P., \& Jaafar, H. (2017). Effect of plasma antenna shape on the antenna performance using plasma computer simulation technology (CST). Journal of Telecommunication, Electronic and Computer Engineering, 9(3-8), 5-9.

Desire, R. A. S. (2018). Comparative Analysis and Design of a Plasma Based Monopole Antenna and a Metal Based Monopole Antenna. 9th International Conference on Information and Communication Technology Convergence: ICT Convergence Powered by Smart Intelligence, ICTC 2018, 1170-1173.

Ghaderi, M., Moradi, G., \& Mousavi, P., (2019). Estimation of Plasma and Collision Frequencies Using Modified Microwave Interferometry Methods for Plasma Antenna Applications. IEEE Transaction on Plasma Science, 47(1), 451-456.

Ja'afar, H., Abdullah, R., Omar, S., Shafie, R., Ismail, N., \& Rustam, I. (2018). Design and development of plasma antenna for wi-fi application. Journal of Fundamental and Applied Sciences, 9(5S), 898.

Ja'afar, H., Ali, M. T., Dagang, A. N., Zali, H. M., \& Halili, N. A. (2014). A reconfigurable monopole antenna with fluorescent tubes by using plasma windowing concepts at 4.9GHz. Advanced Materials Research, 905, 432-435.
Kumar, P., \& Kumar, R. (2018). Study of monopole plasma antenna parameters. Indian Journal of Pure and Applied Physics, 56(3), 238-247.

Li, W., Qiu, J., Lin, S., \& Suo, Y. (2011). Analysis and design of plasma monopole antenna. Proceedings of the 2011 6th International ICST Conference on Communications and Networking in China, CHINACOM 2011, (5), 921-924.

Lieberman, M. A., \& Lishtenberg, A. J. (2005). Principles of Plasma Discharges and Material Processing, $2^{\text {nd }}$ edition. John Wiley \& Sons, Inc.

Lister G. G., \& Coe, S. E. (1993). GLOMAC: A One Dimensional Numerical Model For Steady State Low Pressure MercuryNoble Gas Discharges, Computer Physics Communications, 75(1), pp. 160-184.

Lucas J. (2015). What are Radio Waves. Retrieved from https://www.livescience. com/50399-radio-waves.html, 1 Feb 2020

Madda, D. R., (2017). Plasma Antenna. International Journal of Current Engineering and Scientific Research, 4(11), pp. 34-38.

Qayyum, A., Ikram, M., Zakaullah, M., Waheed, A., Murtaza, G., Ahmad, R., \& Chaudhary, K. A. (2003). Characterization of argon plasma by use of optical emission spectroscopy and Langmuir probe measurements. International Journal of Modern Physics, 17(14), 2749-2759.

Shriwas, R., \& Gulhane, S. (2015). Up gradation of Plasma Antenna by Using Fluorescent Tubes, International Journal of Electronics and Telecommunications, 3(1), 9-16.

Shah, M. S., Saleem, M., Ahmad, R., Zakaullah, M., Qayyum, A., \& Murtaza, G. (2008). Langmuir probe characterization of nitrogen plasma for surface nitriding of AISI-4140 steel. Journal of Materials Processing Technology, 199(1), 363-368. 
